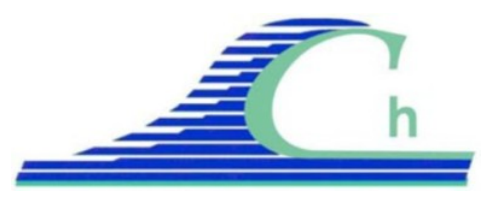

XII ${ }^{\text {èmes }}$ Journées Nationales Génie Côtier - Génie Civil

Cherbourg, 12-14 juin 2012

DOI:10.5150/jngcgc.2012.104-L_ @ Editions Paralia CFL

disponible en ligne - http://www.paralia.fr - available online

\title{
Etude probabiliste des vagues cycloniques le long du littoral Guadeloupéen
}

\author{
Sophie LECACHEUX ${ }^{\mathbf{1}}$, Hélö̈se MULLER ${ }^{\mathbf{1}}$ Rodrigo PEDREROS $^{\mathbf{1}}$, \\ Jérôme THIEBOT ${ }^{2}$, Jehane OURIQUA ${ }^{3}$, Arnaud REVEILLERE ${ }^{1}$
}

\author{
1. BRGM, Service RIS, 3, av. Claude Guillemin, 45060 Orléans Cedex 2, France. \\ s.lecacheux@brgm.fr, h.muller@brgm.fr, r.pedreros@brgm.fr, a.reveillere@brgm.fr \\ 2. ESIX Normandie/LUSAC. Site Universitaire de Cherbourg BP. 78 - 50130 Octeville, \\ France.jerome.thiebot@unicaen.fr \\ 3. Ecole Centrale Nantes, Nantes.France.Jehane.ouriqua@centraliens-nantes.net
}

\section{Résumé :}

La submersion marine liée aux cyclones fait partie des risques majeurs rencontrés dans les Antilles françaises. Une bonne estimation des états de mer extrêmes est un élément primordial pour l'aménagement du littoral et une gestion raisonnée des risques. Dans cette étude, une estimation des périodes de retour des hauteurs de vagues cycloniques en zone côtière a été réalisée sur plusieurs sites du littoral Guadeloupéen. La méthode utilisée comprend (1) la sélection d'évènements pertinents dans la base de données HURDAT (2) la reconstitution des champs de vent pour chaque cyclone à l'aide d'un modèle paramétrique (3) la modélisation des vagues jusqu'en zone côtière (4) une analyse statistique sur la hauteur significative des vagues.

Le système de modélisation a été validé par comparaison avec des données de bouées sur quelques cyclones récents. Les premiers résultats obtenus ont montré une plus forte exposition des sites de la façade Atlantique par rapport à ceux côté mer des Caraïbes. Cependant, les intervalles de confiance calculés restent importants car la base de données historiques utilisée ne fournit qu'un nombre restreint d'évènements utilisables dans l'analyse statistique. Des pistes d'amélioration sont proposées pour des études ultérieures.

Mots-clés : Cyclones - Modélisation - Vagues - Période de retour

\section{Introduction}

La Guadeloupe est l'une des régions françaises où les risques naturels sont les plus importants, les risques volcanique, sismique et cyclonique étant dominants. Les cyclones causent des dégâts via les vents violents, les fortes pluies et la submersion marine, cette dernière ayant joué un rôle prépondérant lors du plus important évènement historique connu, en 1928. Le projet FP7 MATRIX propose de mener une étude comparative des coûts associés aux principaux aléas naturels affectant la Guadeloupe pour une même période de retour. Dans ce cadre, une méthode pour estimer l'aléa submersion marine de manière probabiliste a été mise en place. Cette communication 


\section{Thème 7 - Océanographie opérationnelle et situations extrêmes}

présente la première partie de l'étude sur l'analyse des hauteurs significatives des vagues ainsi que les premiers résultats obtenus. A terme, ces résultats seront couplés à des données de niveau d'eau (marée et surcote atmosphérique) pour étudier l'aléa submersion marine dans sa globalité.

Les modèles atmosphériques et océaniques globaux n'ont pas une résolution suffisante pour reproduire correctement les phénomènes cycloniques ainsi que les vagues et les surcotes induites. Les résultats de simulations rétrospectives et de ré-analyses ne peuvent pas être utilisés pour conduire une analyse statistique. Pour ce type d'aléa complexe, qui dépend d'un nombre important de paramètres (trajectoire et intensité du cyclone, configuration du site local), les méthodes traditionnelles consistent à identifier un ou plusieurs évènements associés à une période de retour donnée, à déplacer leur trajectoire de manière à impacter le plus possible le site étudié, et à calculer les paramètres hydrodynamiques correspondants. Dans cette méthode, la période de retour du cyclone est directement associée à celle des conditions hydrodynamiques locales, ce qui n'est pas toujours vérifié en pratique.

Ici, nous utilisons un autre type de méthode qui consiste à déterminer les caractéristiques locales des vagues générées par un très grand nombre de cyclones afin d'effectuer une analyse statistique directement sur les caractéristiques hydrodynamiques en zone côtière. Ce type d'approche a déjà été utilisé dans plusieurs études, par exemple sur le Queensland (HARDY et al., 2004) ou la ville de New-York (LIN et al., 2010). Ces deux études ont utilisé des bases de cyclones synthétiques, créées à l'aide de modèles statistiques, afin d'augmenter la taille de l'échantillon à simuler et de balayer ainsi un grand nombre de configurations possibles (plusieurs milliers). Cette technique permet d'améliorer la qualité de l'analyse statistique et de calculer des périodes de retour plus importantes mais nécessite le développement d'un modèle statistique complexe ainsi que d'importantes capacités de calcul. Pour notre étude, la méthode a été appliquée en utilisant directement la base de données historiques HURDAT (JARVINEN et al., 1984) qui remonte jusqu'à la moitié du $19^{\text {ème }}$ siècle. Les limites inhérentes à ce choix sont explicitées au paragraphe 2.1 .

La méthode est présentée dans la section 2: nous décrivons la base de données HURDAT, le système de modélisation et la méthode statistique utilisée. La section 3 présente la validation du système de modélisation et les premiers résultats statistiques.

\section{Méthodes}

La méthode mise en place comprend (1) la sélection d'un échantillon de cyclones pertinents en termes de vagues et de surcotes pour la Guadeloupe à partir des "besttracks" de la base de données HURDAT ; (2) la construction des champs de vent pour chaque cyclone avec le modèle paramétrique de HOLLAND (1980); (3) la modélisation des vagues hauturières et côtières; (4) l'extraction des paramètres de vagues en zone côtière autour de l'île et l'analyse statistique en chaque point. 


\section{XII ${ }^{\text {èmes }}$ Journées Nationales Génie Côtier - Génie Civil \\ Cherbourg, 12-14 juin 2012}

\subsection{La base de données HURDAT (National Hurricane Center)}

HURDAT est la base de données officielle pour les cyclones en Atlantique Nord. Elle remonte jusqu'en 1851 et fournit, pour chaque cyclone, une estimation de la position et de l'intensité maximum du vent de surface moyenné sur 1 minute (nommé Vm par la suite) toutes les 6 heures. Les données les plus anciennes sont issues d'observations de navires ou de populations côtières alors que les plus récentes (à partir des années 70) ont été obtenues par analyse de données satellite. La qualité et l'exhaustivité de la base est donc hétérogène dans le temps même si HURDAT est régulièrement mis à jour grâce à des travaux de ré-analyse initiés par LANDSEA et al. (2004) pour corriger les biais et rajouter des évènements. Nous avons choisi de concentrer notre étude sur le $20^{\text {ème }}$ siècle (de 1910 à 2009) car la densité de la navigation et de la population laissent présumer d'une assez bonne représentation des cyclones les plus importants (LANDSEA, 2007). $\mathrm{Au}$ total, 291 cyclones ont été sélectionnés, principalement sur des critères géographiques (passage d'au moins $24 \mathrm{~h}$ dans le domaine $5^{\circ} \mathrm{N}-30^{\circ} \mathrm{N}$ et $70^{\circ} \mathrm{W}-45^{\circ} \mathrm{W}$ ). Pour ces cyclones, les vents fournis dans HURDAT ont été comparés avec les données $\mathrm{H}^{*}$ Wind (NOAA) qui proposent, pour les cyclones à partir des années 90 , des champs de vent ré-analysés à partir de toutes les observations disponibles (POWELL et al., 1998). Cette comparaison a montré une surestimation d'environ $10 \%$ des vents HURDAT, quelle que soit la catégorie du cyclone (avec un coefficient de détermination $\mathrm{R}^{2}$ d'environ 0.93 ). Aussi, une correction de $10 \%$ a été appliquée à l'ensemble de la base.

\subsection{Création des champs de vent paramétriques}

Pour chaque cyclone, les paramètres sont interpolés toutes les demi-heures, puis les champs de vent sont calculés à chaque pas de temps sur une grille de $0,1^{\circ}$ de résolution (voir exemple sur la figure 1). La formule de HOLLAND (1980), permet de calculer le profil de la vitesse radiale du vent de surface $(\mathrm{Vr})$ selon la distance $r$ à l'œil avec l'équation (1):

$V_{r}=\sqrt{B \frac{\left(P_{n}-P_{c}\right)}{\rho_{a}} \times\left(\frac{R_{m}}{r}\right)^{B} \times \exp \left(-\left(\frac{R_{m}}{r}\right)^{B}\right)+\frac{r^{2} f^{2}}{4}}-\frac{r f}{2} \quad \Rightarrow \quad B \approx \frac{V_{m}{ }^{2} \rho_{a} e}{\left(P_{n}-P_{c}\right)}$

où $\mathrm{f}$ est la force de Coriolis et $\rho \mathrm{a}$, la masse volumique de l'air $\left(\sim 1.15 \mathrm{~kg} \cdot \mathrm{m}^{-3}\right)$.

La base HURDAT ne renseignant que le paramètre $V_{m}$ (et la pression au centre $P_{c}$ pour les cyclones les plus récents), les autres paramètres ont été fixés à partir de valeurs de référence pour l'Atlantique nord d'après HOLLAND (1980 et 2008): $R_{m}$, le rayon de vent maximum, est fixé à $30 \mathrm{~km}$, et $P_{n}$, la pression de surface ambiante, est fixée à $1015 \mathrm{hPa}$. Le paramètre $B$, qui représente la forme générale du profil, est calculé à partir de la relation entre $V_{m}$ et $P_{c}$, ou est fixé à 1,26 (valeur médiane quand le profil est ajusté avec les vents de surface moyennés sur $1 \mathrm{~min}$ ) quand $P_{c}$ n'est pas renseigné. 


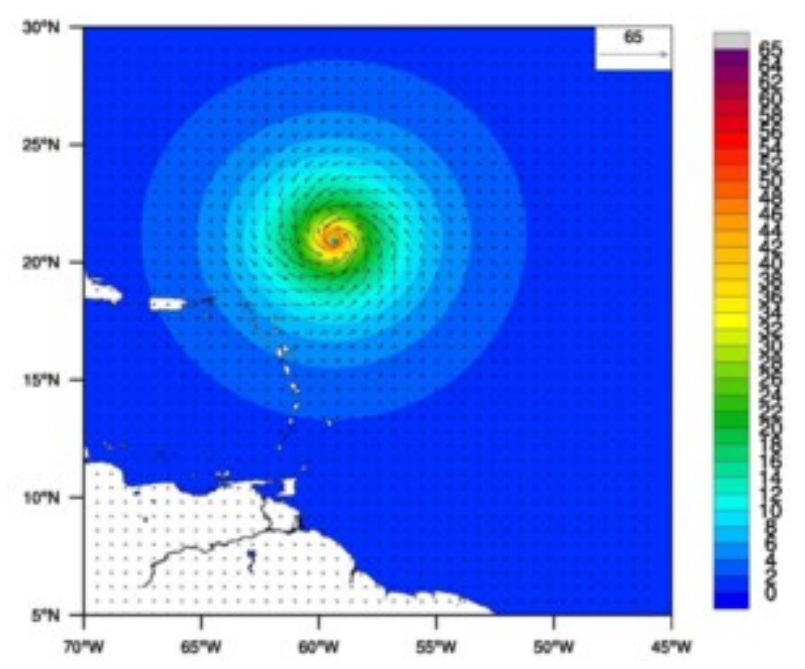

Figure 1. Exemple de champ de vent (m/s) créé pour le cyclone Bill (le 20/08/09 5h).

\subsection{Le système de modélisation}

La modélisation des vagues cycloniques s'appuie sur l'utilisation conjointe des modèles de propagation des vagues WW3 (TOLMAN, 2008) pour les vagues hauturières, et SWAN (BOOIJ et al., 2004) en zone côtière, avec une série de trois emboîtements (figure 2). Les différents modèles sont forcés uniquement avec les champs de vent paramétriques calculés précédemment moyennés sur $10 \mathrm{~min}$. Les séries temporelles de vagues (Hs, Tp, Dp) sont extraites pour les 291 cyclones sur des points côtiers à différentes profondeurs allant de $20 \mathrm{~m}$ à $50 \mathrm{~m}$ le long de la côte guadeloupéenne.
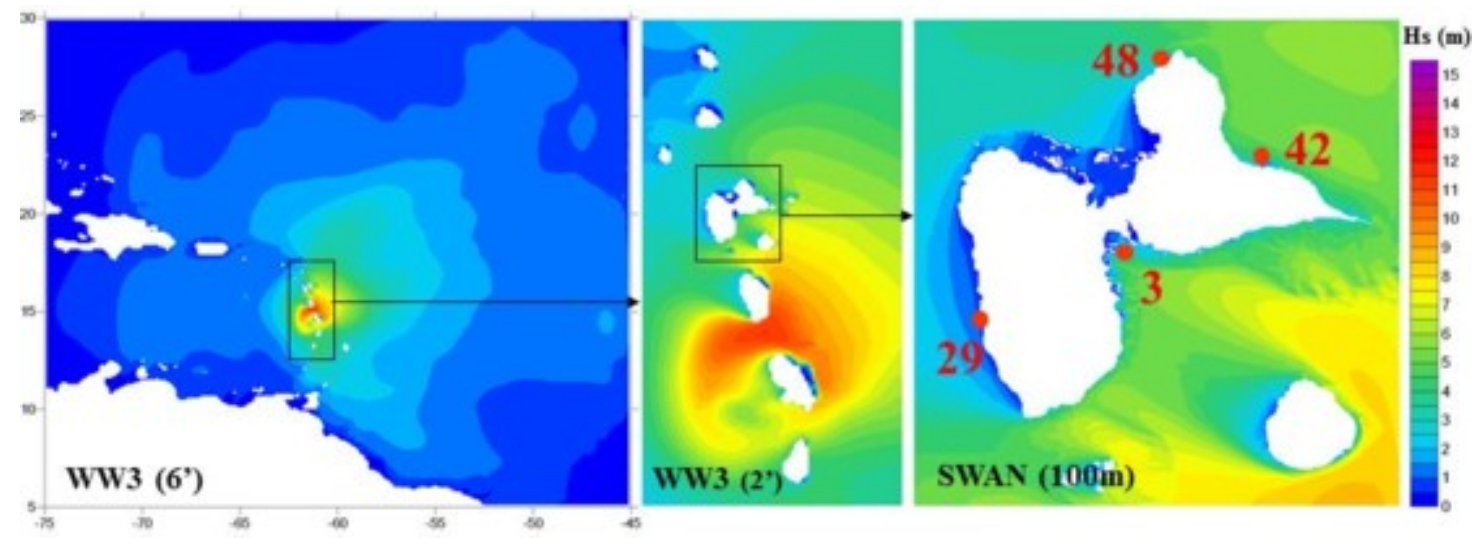

Figure 2. Exemple de simulation pour le cyclone Dean avec les modèles WW3 et SWAN et points de sortie sur l'isobathe à 20 m utilisés pour illustrer l'analyse statistique (3.2).

\subsection{Analyse statistique}

L'analyse statistique sur la hauteur significative des vagues a été conduite à l'aide de fonctions de la boîte à outils WAFO de Matlab (BRODTKORB et al., 2000). Chaque cyclone est représenté par le pic de hauteur significative qui a été simulé sur le point 


\section{XII ${ }^{\text {èmes }}$ Journées Nationales Génie Côtier - Génie Civil \\ Cherbourg, 12-14 juin 2012}

considéré. Tout d'abord, les évènements indépendants sont sélectionnés par la méthode des seuils (Peaks Over Threshold, POT). Puis, une loi Generalized Pareto Distribution (GPD) est ajustée avec la méthode de maximum de vraisemblance.

\section{Résultats intermédiaires pour les vagues}

\subsection{Validation}

Pour la validation des simulations, les bouées hauturières du NDBC (NOAA) 41046, 41044, 42059, 42060, 41040, les bouées de Météo France 41100 et 41101 ainsi que la bouée CANDHIS côtière 97103 à la Pointe de la Grande Vigie ont été utilisées (figure 3 , gauche). Ces observations sont pour la plupart récentes, ce qui limite le nombre de cyclones utilisables pour la validation. Deux cyclones ont été testés : Dean (2007), et Bill (2009). De manière générale, la comparaison entre les mesures et les simulations donne des résultats satisfaisants au moment du pic des vagues quand la trajectoire du cyclone passe près de la bouée car la plupart de l'énergie des vagues est générée par le cyclone lui-même. Plus la bouée est éloignée de la trajectoire, plus les hauteurs de vagues sont sous-estimées car celles-ci sont en partie générées par les vents synoptiques non pris en compte dans le modèle paramétrique. La fusion du vent GFS (NOAA) avec le vent paramétrique, quand celui-ci devient inférieur à $10 \mathrm{~m} / \mathrm{s}$, permet de retrouver des hauteurs comparables aux enregistrements (voir l'exemple pour Bill sur la figure 3, droite). Le pic de la hauteur des vagues liées au cyclone (ce qui nous intéresse dans cette étude) est donc correctement reproduit. Toutefois, on remarque une surestimation générale des Hs à gauche de la trajectoire des cyclones (dans le sens de propagation). En effet, le modèle paramétrique utilisé reconstitue un cyclone symétrique alors qu'en réalité le vent n'est pas réparti uniformément autour de l'œil à cause de nombreux phénomènes dont la vitesse de translation, qui a tendance à accélérer la vitesse du vent à droite de la trajectoire et à la diminuer à gauche de celle-ci (pour l'hémisphère nord). Par exemple, pour le cyclone Dean, on note une erreur relative sur Hs de moins de 3\% au moment du pic pour les bouées 41100 et 41040 mais une surestimation d'environ $30 \%$ au niveau de la bouée 42059 . Outre les simplifications inhérentes au modèle, cette différence peut également s'expliquer par la détermination de certains paramètres avec des valeurs moyennes issues de la littérature faute de données dans HURDAT. Par exemple, le rayon de vent maximum est fixé à $30 \mathrm{~km}$ alors que celui-ci est très variable d'un cyclone à un autre. Cette simplification peut avoir un effet très important sur les vagues simulées, notamment très proches de l'œil. La validation du système de modélisation des vagues a donc mis en avant soit une représentation correcte du pic de hauteur significative des vagues cycloniques, soit une surestimation dans certain cas. Ces résultats s'inscrivent dans une approche conservative adaptée pour une étude de risques. 

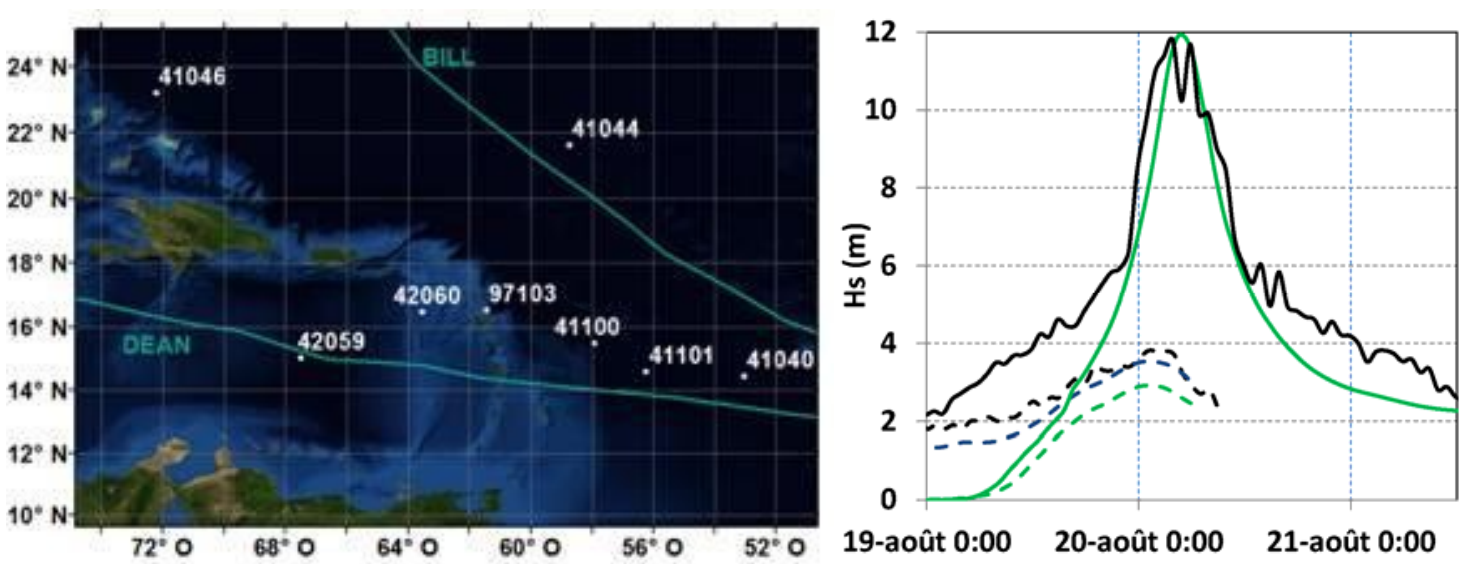

Figure 3. Gauche : Trajectoires des cyclones Bill et Dean et position des bouées utilisées pour la validation. Droite : comparaison des Hs mesurées par les bouées

97103 (pointillés) et 41044 (trait plein) avec les Hs simulées avec les vents paramétriques seuls (vert) et fusionnés avec GFS (bleu) pour le cyclone Bill.

\subsection{Analyse statistique}

L'analyse statistique a été effectuée sur différents points autour de la Guadeloupe en fixant un seuil de $\mathrm{Hs}=1 \mathrm{~m}$ pour la sélection des évènements. Les résultats sont présentés pour 4 points situés sur l'isobathe $20 \mathrm{~m}$ (figure 4). Quelle que soit la localisation des points autour de l'île, le nombre d'évènements considérés s'est avéré suffisant pour ajuster une loi GPD. Tous les événements sont compris dans les intervalles de confiance à $95 \%$. De manière générale, la façade Atlantique du littoral est plus exposée aux houles extrêmes que la partie orientée vers la mer des Caraïbes tant sur le nombre d'évènements que sur leur intensité. En effet, les cyclones, qui sont précédés de la houle cyclonique, traversent l'arc Antillais d'est en ouest dans la majorité des cas. La valeur centennale de Hs est estimée à environ $8 \mathrm{~m}$ vers Le Moule (point 42) et $9 \mathrm{~m}$ au niveau de la pointe de la Grande Vigie (point 48), alors qu'elle est plutôt de l'ordre de $5 \mathrm{~m}$ au niveau de la côte sous-le-vent (point 29). Pointe-à-Pitre est exposé à des vagues moins fortes de par sa configuration en fond de baie, qui constitue un filtre naturel, et la protection offerte par Les Saintes et Marie-Galante au sud de l'île.

Les intervalles de confiance calculés sont assez larges, surtout pour les points côté façade Atlantique (42 et 48) pour lesquels on note une moins bonne adéquation entre les lois empiriques et théoriques. Dans ce secteur, le nombre d'évènements utilisés est insuffisant pour estimer des périodes de retour avec certitude.

\section{Conclusions et perspectives}

Ces travaux ont permis d'obtenir une première estimation des périodes de retour des hauteurs significatives des vagues cycloniques en zone côtière pour la Guadeloupe. Il faut toutefois prendre ces résultats avec précaution en raison des limitations inhérentes à 


\section{XII ${ }^{\text {èmes }}$ Journées Nationales Génie Côtier - Génie Civil \\ Cherbourg, 12-14 juin 2012}

la base de données utilisée (fenêtre temporelle courte et nombre restreint d'évènements pris en compte, incertitudes pour les cyclones les plus anciens) et aux simplifications liées au modèle paramétrique de vent. L'étude des niveaux d'eau, effectuée avec la même base de données historiques, est en cours de réalisation.

Ce travail pourrait être amélioré avec l'utilisation d'une base de cyclones synthétiques, comme celle d'EMANUEL et al. (2006), qui fournit un nombre de trajectoire très important. Ceci permettrait de considérer beaucoup plus de configurations possibles et rendrait plus robuste l'analyse statistique en réduisant les intervalles de confiance. De plus, avec un nombre d'évènements accru, un travail par probabilités conjointes sur les vagues et les niveaux d'eau pourrait être envisagé.
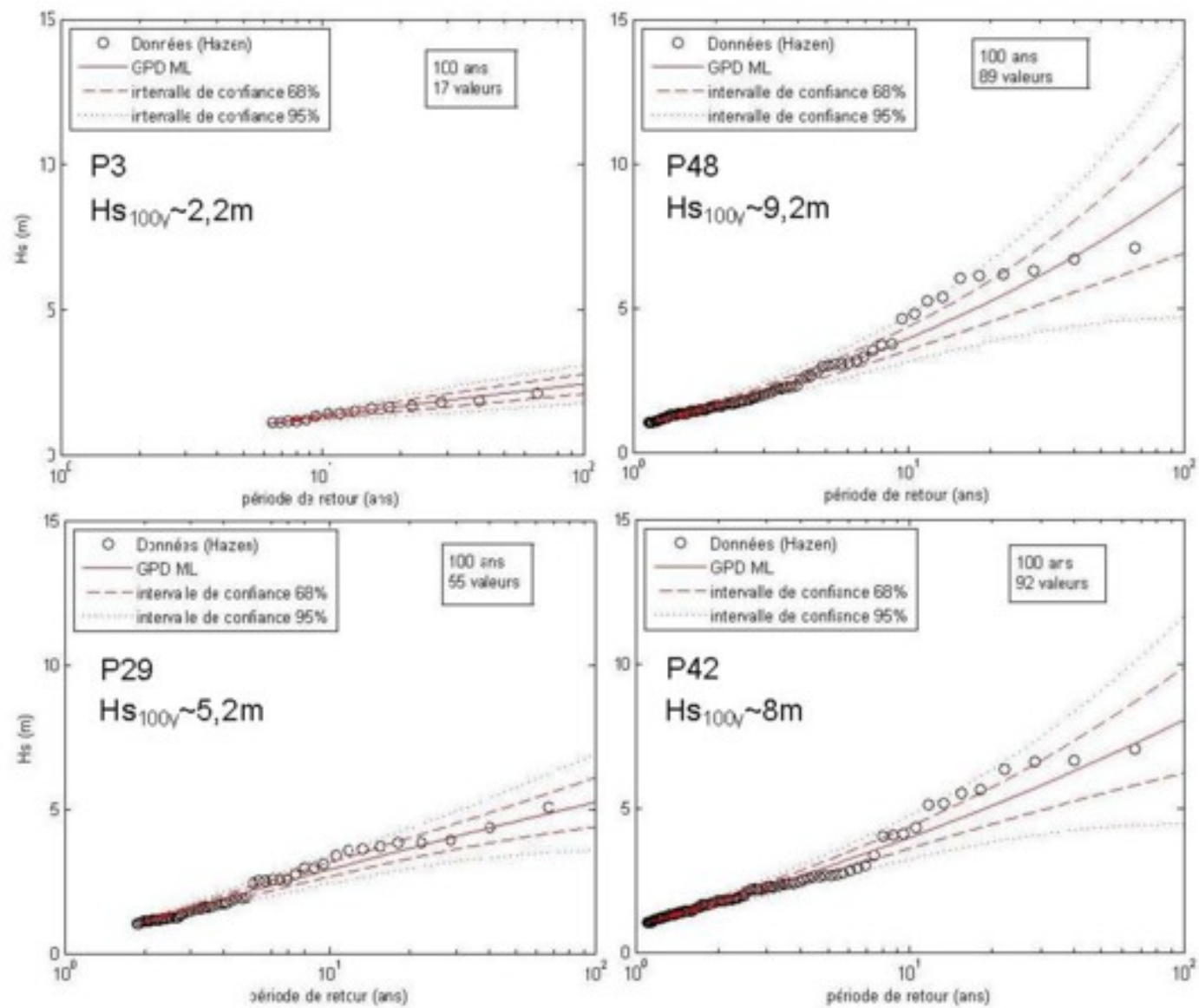

Figure 4. Résultat de l'analyse statistique pour les points 3, 29, 42 et 48 de la figure 2.

\section{Remerciements :}

Cette étude a été financée par le projet FP7 MATRIX et le projet cadre de recherche et développement RISCOTE du BRGM. 
Thème 7 - Océanographie opérationnelle et situations extrêmes

\section{Références bibliographiques}

BOOIJ N., HAAGSMA IJ.G., HOLTHUIJSEN L.H., KIEFTENBURG AT.M.M, RIS R.C., VAN DER WESTHUYSEN A.J., ZIJLEMA M. (2004). Swan Cycle III version 40.41, User's Manual, 115 p.

BRODTKORB P.A., JOHANNESSON P., LINDGREN G., RYCHLIK I., RYDEN J., SJÖ E. (2000). WAFO - a Matlab toolbox for analysis of random waves and loads, in: Proceeding of the 10th Int. Offshore and Polar Eng. Conf., Seattle, USA, Vol. III, pp 343-350.

EMANUEL K., RAVELA S., VIVANT E., RISI C. (2006). A statistical deterministic approach to hurricane risk assessment. Bull. Am. Meteorol. Soc., 87, pp 299-314. doi:10.1175/BAMS-87-3-299

HARDY T., MASON L., ASTORQUIA A., HARPER B. (2004). Queensland climate change and community vulnerabilty to tropical cyclones: Ocean hazards assessement, stage 2. Tropical cyclones-indiced water level and waves: Hervey Bay and Sunshine Coast.

HOLLAND G. (1980). An analytic model of the Wind and Pressure Profiles in Hurricanes. Monthly Weather Review, Vol. 108, pp 1212-1218. doi:10.1175/15200493(1980)108<1212:AAMOTW>2.0.CO;2

HOLLAND G. (2008). A revised hurricane pressure-wind model._Monthly Weather Review, vol. 136, pp 3432-3445. doi:10.1175/2008MWR2395.1

JARVINEN B.R., NEUMANN C.J., DAVIS M.A.S. (1984). A tropical cyclone data tape for the North Atlantic Basin, 1886-1983: Contents, limitations, and uses. NOAA Technical Memorandum NWS NHC 22, Coral Gables, Florida, 21 p.

LANDSEA C.W. (2007). Counting Atlantic tropical cyclones back to 1900. EOS, Trans. American Geophysical Union, Vol. 88 (18), pp 197-202. doi:10.1029/2007EO180001

LANDSEA C. W., ANDERSON C., CHARLES N., CLARK G., DUNION J., FERNANDEZ-PARTAGAS J., HUNGERFORD P., NEUMANN C., ZIMMER M. (2004). The Atlantic hurricane database re-analysis project: Documentation for the 1851-1910 alterations and additions to the HURDAT database. Hurricanes and Typhoons: Past, Present and Future, R. J. Murname and K.-B. Liu, Eds., Columbia University Press, pp 177-221.

LIN N., EMANUEL K.A., SMITH J.A., VANMARCKE E. (2010). Risk assessment of hurricane storm surge for New York City. J. Geophys. Res., 115, D18121, doi:10.1029/2009JD013630

POWELL M.D., HOUSTON S.H., AMAT L.R., MORISSEAU-LEROY N. (1998): The HRD real-time hurricane wind analysis system. J. Wind Engineering and Indust. Aerodyn. Vol. 77\&78, pp 53-64.

TOLMAN H.L. (2008). A mosaic approach to wind wave modeling. Ocean Modelling, 25, pp 35-47. doi:10.1016/j.ocemod.2008.06.005 